\title{
32nd ANNUAL SASKATCHEWAN CHRISTMAS BIRD COUNT 1973
}

\author{
Compiled by MARY I. HOUSTON*
}

Cold weather and much deeper snow than usual made bird counting more difficult this year. A number of areas were covered by snowshoers or skiers where cars could not operate. Consequently, it was most encouraging to note that counters were out in 42 areas (Fig. 1), and observed total of 67 species plus 4 other species seen during count period but not seen on count days. This total of species has been exceeded only once - in 1962 when 68 species with 8 additionals were recorded.

One new species were added to the all-time total. A Black Duck was reported from Regina. A Mountain Bluebird seen at Fort Qu'Appelle during the count period (though not on count day) adds a new "additional" to the grand total. This total is now 116 species and 7 additionals.

There were no major increases or decreases in species occurrences. Of interest was the fact that Golden Eagles were not seen on count day (and only once during count period) for the first time in 10 years. As in 1971, Gyrfalcons were reported from 2 localities. This is the third year of the 32 in which counts have been taken that this species has been reported.

I. BANGOR. Dec. 26; temp. $19^{\circ}$, around yard and feedstacks; 8 species, 45 individuals. - Mrs. A. Thompson.

2. BIGGAR. Dec. $24 ; 3 \mathrm{mi}$. in 3 hrs. on foot and $15 \mathrm{mi}$. in $1 / 2 \mathrm{hr}$. by car, temp. $20^{\circ}$, fog patches, calm; 11 species, 496 individuals. (Add: Rusty Blackbird, 2, Dec. 23) - Donald Renaud, Wayne Renaud (compiler).

3. BORDEN. Dec. 20;79 mi. by car in 7

*863 University Drive, Saskatoon, Sask. hrs. and $3 \mathrm{mi}$. on foot in $1-1 / 2$ hrs., temp. $9^{\circ}$ to $22^{\circ}$, partly cloudy, wind SE $0-13 \mathrm{mph}, 8$ inches of snow; 13 species, 1796 in dividuals. - Ron Bobowski, Vi Harper Vic Harper, John Shadick, Stan Shadick (compiler).

4. BROADVIEW. Dec. 30; $123 \mathrm{mi}$. by car in 6 hrs., temp. $-30^{\circ}$, clear, wind light; 10 species, 529 individuals. (Add: Rock Dove, 12, Dec. 29; Hairy Woodpecker, 1, Dec. 29) - David Chaskavich, Don Weidl.

5. CARROT RIVER. Dec. 27; $60 \mathrm{mi}$. by car in $1-1 / 2$ hrs., temp. $15^{\circ}$, calm: 7 species 12 individuals. - Barbara and Ronald Hooper.

6. DALMENY. Dec. $26 ; 44-3 / 4$ miles in 23 hrs. on skis, temp. $15^{\circ}$, wind NW $13 \mathrm{mph}$, overcast, light snow; 12 species, 747 individuals. (Add: Starling, 9, Dec. 28) Brian Sperling, Gilbert Sperling (cocompiler), Lorne Sperling, Lloyd Sperling (co-compiler).

7. DILKE. Dec. 27: $23 \mathrm{mi}$. by car and $1 \mathrm{mi}$ by foot and sleigh at chores, temp. 0 to $20^{\circ}$, overcast to sunny, wind SW. changing to NW, rising from light to $15 \mathrm{mph} ; 6$ species 164 individuals. (Add: Snow Bunting, Dec. 22, 26 and 28). - J. B. Belcher.

8. ENDEAVOUR. Dec. 26; around farmyard and $3 \mathrm{mi}$. on foot, temp. $10^{\circ}$, overcast with light snow; 10 species, 250 individuals. (Add: Great Horned Owl, 1, Dec. 24; Blue Jay, 3, Dec. 31; Rusty Blackbird, 1, Dec. 27). - William Haras.

9. FILLMORE. Dec. $27 ; 25 \mathrm{mi}$. by car, $3 / 4$ mi. on foot in $3-1 / 2 \mathrm{hrs}$., temp. $10^{\circ}$, partly cloudy, wind 15-20 mph with drifting snow, 2-1/2 ft. snow: 7 species, 683 individuals. (Add: Sharp-tailed Grouse, 8, Dec. 28; Snowy Owl, 1, Dec. 23). - Larry and Marie Wiggins, Michael and Michelle Wiggins.

10. FORT QU'APPELLE. Dec. 29; $96 \mathrm{mi}$. by car in 9 hrs., temp. $-20^{\circ}$ to $-15^{\circ}$, wind NW 5-9 mph, 12-1/2 in. snow; 20 species, 445 individuals. (Add: Merlin, 1, Dec. 17; Ruffed Grouse, 1, Dec. 21 and 2, Dec. 27; Ring-necked Pheasant, 1, Dec. 26: Snowy Owl, 1, Dec. 21; White-breasted Nuthatch, 1, Dec. 22 and 28; Mountain Bluebird, 1, 


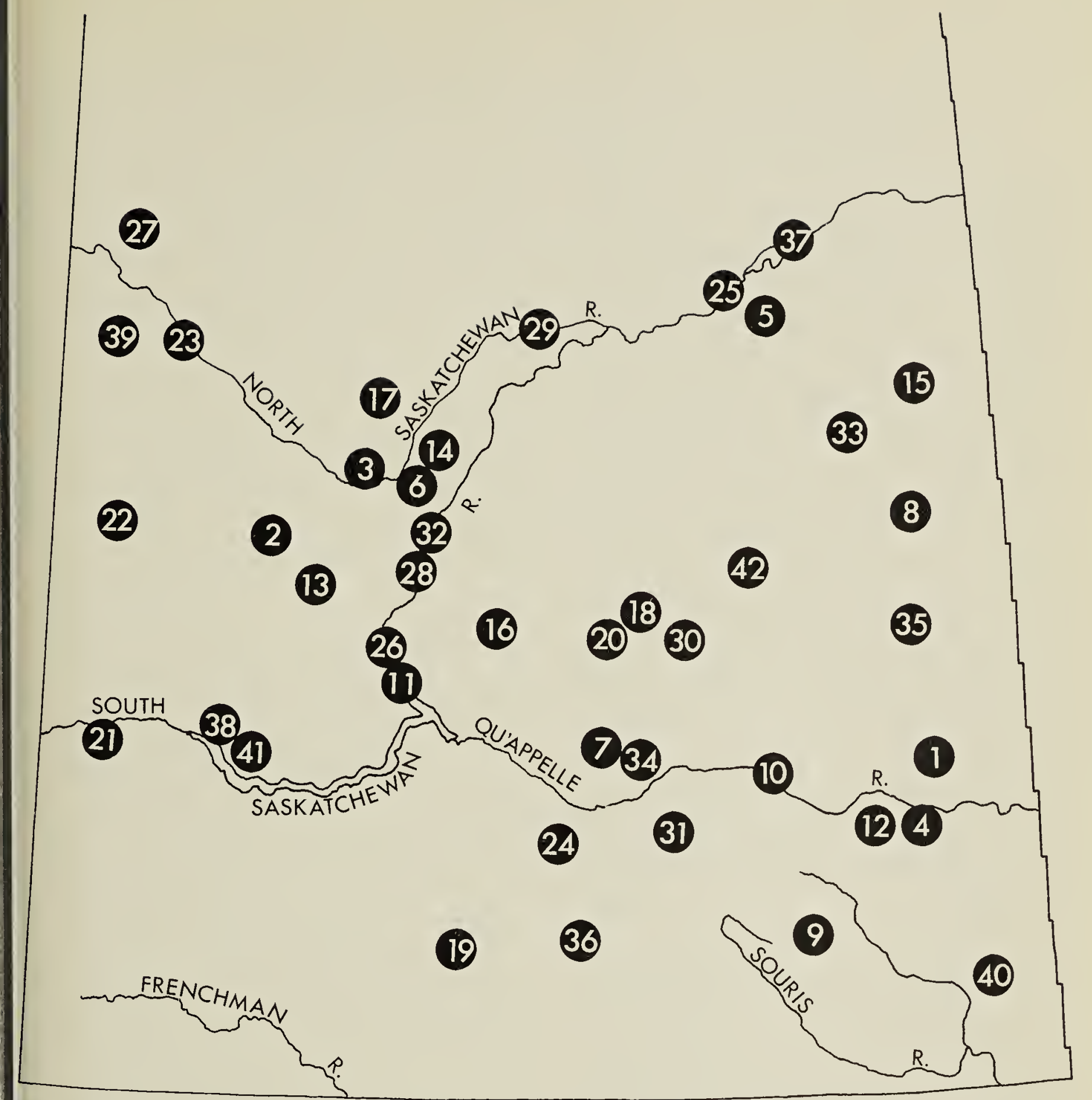

Figure 1. Locations of Saskatchewan Christmas Bird Counts, 1973. (See text for identification of numbers.)

Dec. 26; Rusty Blackbird, I, Dec. 27 and 5. laily to Dec. 21; Evening Grosbeak, 2, pec. 26). - E. Manley Callin, Brian Gladvell, Mrs. William Gray, Wanda and Paul Hanley, Ron and Don Hooper, Inez and oseph Kralkay, Shirley Spidla, Mrs. Cy Villiams and Cynthia Williams, Kay and Bernard de Vries (compilers) - Members of the Fort Qu'Appelle Naturalists Society.

1. GARDINER DAM. Dec. 27; $9 \mathrm{mi}$. on oot in $4 \mathrm{hrs}$. and 30 . mi. by car in $1-1 / 2$ irs., temp. $10^{\circ}$, clear, wind NW $8 \mathrm{mph} ; 17$ pecies, 647 individuals. - Wayne Harris, heila Lamont, Don Renaud, Wayne Renaud (compiler).

2. GRENFELL. Dec. 24; around yard and hort drive, temp. $26^{\circ}$, calm, 12 in. snow; 5 species, 206 individuals. (Add: Ringnecked Pheasant, 1, Dec. 20; Gray Partridge, 6, Dec. 31; Snowy Owl, 1, Jan. 2; Black-billed Magpie, 1, Jan. 2). - Betty Hubbard.

13. HARRIS. Dec. 22; $125 \mathrm{mi}$. in $12 \mathrm{hrs}$. by car, $24 \mathrm{mi}$. in $19 \mathrm{hrs}$. on foot, $3 \mathrm{mi}$. in $2 \mathrm{hrs}$. by snowmobile, temp. $25^{\circ}$ to $28^{\circ}$, calm, fog patches; 17 species, 2515 individuals. (Add: Merlin, 1, Dec. 2l; Evening Grosbeak, 2, Dec. 23). - Mark Abley, Ron Bobowski, Tom Donald, Bob Godwin, Allan Moulin, Lynn Oliphant, Don Renaud, Wayne Renaud (compiler), Stan Shadick.

14. HEPBURN. Dec. $25 ; 6$ species, 93 individuals. - Margaret, Philip, Phyllis and 
Pied-billcd Grebe

Mutc Swan

Canada Goosc

Mallard

Black Duck

Gadwall

Pintail

Redhead

Canvasback

Lesser Scaup

Common Goldeneye

Bufflehead

Ruddy Duck

Common Mcrganser

Red-br. Merganscr

Goshawk

Bald Eagle

Gyrfalcon

Prairie Falcon

Merlin

Ruffed Grouse

Sharp-tailed Grouse

Ring-ncck. Pheasant

Gray Partridge

Amcrican Coot

Rock Dove

Great-horncd Owl

Snowy Owl

Hawk Owl

Grcat Gray Owl

Long-eared Owl

Short-eared Owl

Common Flicker

Pileated Woodpccker

Hairy Woodpecker

Downy Woodpecker

N. 3-t. Woodpecker

Horned Lark

Gray Jay

Bluc Jay

Black-billed Magpic

Common Raven

Bl.-cap. Chickadee

Borcal Chickadee

W-breasted Nuthatch

R-breasted Nuthatch

Brown Creeper

Robin

Bohemian Waxwing

Cedar Waxwing

Northern Shrike

Starling

House Sparrow

Rusty Blackbird

Brewer`s Blackbird

Common Grackle

Evening Grosbeak

Purple Finch

Pinc Grosbcak

Hoary Redpoll

Common Redpoll

Pine Siskin

W-winged Crossbill

Dark-eyed Junco

Tree Sparrow

W-throated Sparrow

Snow Bunting

\begin{tabular}{|l|l|l|l|l|l|l|l|}
\hline & & & & & & \\
\hline
\end{tabular}

$+\frac{1}{2}+$




\section{Tena Siemens}

15. HUDSON BAY. Dec. 29;60 mi. by car, temp. - $15^{\circ}$; wind NE $15 \mathrm{mph}$; 11 species, 102 individuals. - Darren Hayes, Dwight Hayes, Les Hayes, Eldon Thorson.

16. KENASTON. Jan. 2; within $2 \mathrm{mi}$. of farmyard, temp. $0^{\circ}$, wind NW $10 \mathrm{mph}, 10$ in. snow: 5 species, 100 individuals. (Add: Sharp-tailed Grouse, 16, Jan. 3; Downy Woodpecker, 1, Dec. 21; Bohemian Waxwing. 15, Dec. 21, 22; Common Redpoll, 1, Dec. 22, 23, 24; Snow Bunting, 25, Dec. 24). - P. Lawrence Beckic.

17. KRYDOR. Dec. 26:24 mi. by car and 3 mi. on skis in 3 hrs., temp. $20^{\circ}$, wind WSW $7 \mathrm{mph}$, overcast with some light snow: 3 species, 18 individuals - Robin and Keith Bracken, Frank, Louise, Molly and Ray Denson, Barry and Jane Findlayson, Charles, John and Peggy Hall, Susan Lamers, John and Renee Merz, Alan and Dolores Reid.

18. KUTAWAGAN LAKE (centered 12 mi. N. of Semans). Dec. 29;30 mi. by car in 4 hrs., 2 mi. on snowshoes in 1 hr., temp. $-28^{\circ}$ to $-25^{\circ}$, clear, wind $0-5 \mathrm{mph}, 15$ in. now: 10 species, 348 individuals. Wayne Harris, Sheila Lamont.

19. LAFLECHE. Dec. 26; temp. $20^{\circ}$, light wind, 10 in. snow; 9 species, 197 individuals. (Add: Golden Eagle, 1, Dec. 19; Snowy Owl, 1, Dec. 27), - C. H. Shulver.

\section{LAST MOUNTAIN LAKE} (management unit and immediate area). Dec. $15 ; 62 \mathrm{mi}$. by car in $5 \mathrm{hrs}$. and $6 \mathrm{mi}$. on foot in $2 \mathrm{hrs}$., temp. $-21^{\circ} \mathrm{to}-9^{\circ}$, sunny, wind ESE 7-15 mph, 15 in. snow; 9 species, 200 Individuals. - Greta Harris, Wayne Harris, Sheila Lamont.

21. LEADER. Jan. 2; temp. $12^{\circ}$, snowing, 1 foot of snow; 4 species, 31 individuals. Daisy D. Meyers.

22. LUSELAND. Dec. 22; $8 \mathrm{mi}$. by car and If mi. by snowshoe in $4 \mathrm{hrs}$., temp. $16^{\circ}$ to $122^{\circ}$, dense fog, 1 foot of snow; 9 species, 119 individuals (Add: Snowy Owl, 1, Dec. 27: Downy Woodpecker, 1, Dec. 27; Horhed Lark, 6, Dec. 31). - K. B. Finley, K. J. Finley, B. Holton.

23. MAIDSTONE FERRY (21 mi. north of Maidstone). Dec. 22; 36 mi. by car in 2 hrs. and $7 \mathrm{mi}$. on snowshoes in $3-1 / 2 \mathrm{hrs}$., temp. $10^{\circ}$ to $15^{\circ}$, cloudy with heavy fog, wind $E$ ) -5 mph, 15 in. snow; 15 species, 559 individuals. (Add: Rock Dove, 2, Dec. 23; nnowy Owl, 1, Dec. 26; Blue Jay, 1, Dec. 24; Bohemian Waxwing, 20, Dec. 24; Snow Bunting, 1, Dec. 24, 50, Dec. 25 and 13 , Dec. 26). - Wayne Harris, Sheila Lamont, Tommy Lamont, Jean McPherson.

24. MOOSE JAW. Dec. 26;80 mi. by car and $4 \mathrm{mi}$. on foot, tcmp. $-16^{\circ}$, wind $10 \mathrm{mph}$, overcast, 2 fect of snow: 17 species, 1,086 individuals. (Add: White-winged Crossbill, 4, Dec. 21). - Doug Francis, Ruth Hilling. Pat Kcrn, Lcith Knight (compiler), Sid and Mickey Lane, Moray Lewis, Dave Robinson, James Roe, Jennifer Roe and Inez Simmonds.

25. NIPAWIN. Dec. 29; temp. $-20^{\circ}$, heavy hoarfrost; 8 species, 108 individuals. (Add: Pileated Woodpecker, 1, Dec. 25; Hairy Woodpccker, 1, Dcc. 26; Downy Woodpecker, 1, Dcc. 25; Black-backed Threetoed Woodpecker, 1, Dec. 26; Blackcapped Chickadee, 10, Dec. 26). - Robert Moorc, Stan and Gladys Riome.

26. OUTLOOK. Dec. 22; around the town, temp. $-20^{\circ}$, much snow; 2 species, 81 individuals. (Add: Black-billed Magpie, 2, Jan. I). - Harold Kvinge.

27. PARADISE HILL-MINISTIKWAN LAKE. Dec. 24; $71 \mathrm{mi}$. by car in $5 \mathrm{hrs}$. and $3 \mathrm{mi}$. on snowshoes in $1-1 / 2 \mathrm{hrs}$, temp. $10^{\circ}$ to $20^{\circ}$, foggy until noon, then mostly clear, wind SE $5 \mathrm{mph}, 12$ in. snow; 15 species, 319 individuals. - Wayne Harris, Sheila Lamont.

28. PIKE LAKE. Dec. 29; $63 \mathrm{mi}$. by car in 6 hrs., $1 / 2 \mathrm{mi}$. on foot in $1 / 2 \mathrm{hr} ., 1 \mathrm{mi}$. on skis in $1 \mathrm{hr}$., temp. $-16^{\circ} \mathrm{to}-30^{\circ}$, wind $6-11$ mph SW and NW; 14 species, 782 individuals. - Margaret and Ed Driver, Madeleine and Bernard Gollop, Laura Hoyt.

29. PRINCE ALBERT. Dec. $31 ; 8$ species, 39 individuals. - Christie Aschim.

30. RAYMORE. Dec. 28; $67 \mathrm{mi}$. by car in 8 hrs. and $5 \mathrm{mi}$. on snowshoes in $4-1 / 2 \mathrm{hrs} ., 1$ hr. at feeder, temp. $-17^{\circ} \mathrm{t}()-4^{\circ}$, mainly clear with occasional heavy fog patches, wind NW 0-12 mph, 25 in. snow: 17 species, 1300 individuals. (Add: Downy Woodpecker, 1, Dec. 30). - Chas., Greta and Wayne Harris, Sheila Lamont.

31. REGINA. Dec. 26: $254 \mathrm{mi}$. by car in 80 hrs., $40 \mathrm{mi}$. on foot in 32 hrs., 24 hrs. at feeders, temp. $5^{\circ}$ to $17^{\circ}$, overcast with light snow in A.M. mostly cloudy in P.M., wind SE 4 mph to NW 9 mph, 26 in. snow; 40 species, 4,925 individuals.-Gary Anweiler, Jessic Bailey, Fred Bard, Gordon Barr, Kcith Barr, Margaret Belcher (compiler), Tom Beveridge, Al and Betty Binnic, Joanne Boychuk, Tom Burns, Elizabeth Cruickshank, Dick and Maurcen Du Wors, George Ferguson, Bill Freeman, Wayne Gemmell, Valerie Harrison, Dwaync Harty, Jim Hines, Jim and Shirley Jowsey, Ernie Kassian, Darlene Kauk, David Kelly, Bob Krcba, Shirley Larmour, Georgc Ledingham, Christine MacDonald, Mr. and Mrs. J. F. McKay, Juan Martincz, 


\begin{tabular}{|c|c|c|c|c|c|c|c|c|c|c|c|c|c|c|}
\hline Pied-billed Grebe & & & & & & & & & & & & & & \\
\hline Mute Swan & & & & & & & & & & & & & & \\
\hline Canada Goose & & & & & & & & & & & & & & \\
\hline Mallard & & & & & & 1 & & & & & & & & \\
\hline Black Duck & & & & & & & & & & & & & & \\
\hline Gadwall & & & & & & & & & & & & & & \\
\hline Pintail & & & & & & & & & & & & & & \\
\hline Redhead & & & & & & & & & & & & & & \\
\hline Canvasback & & & & & & & & & & & & & & \\
\hline Lesser Scaup & & & & & & & & & & & & & & \\
\hline Common Goldeneye & & & & & & & & & & & & & & \\
\hline Bufflehead & & & & & & & & & & & & & & \\
\hline Ruddy Duck & & & & & & & & & & & & & & \\
\hline Common Merganser & & & & & & & & & & & & & & \\
\hline Red-br. Merganser & & & & & & & & & & & & & & \\
\hline Goshawk & & & & & & & & & 1 & & & & 1 & \\
\hline Bald Eagle & 1 & & & & & & & & & & & & & \\
\hline Gyrfalcon & & & & & & & & & & & & & & \\
\hline Prairie Falcon & & & & & & & & & & & & & & \\
\hline Merlin & & & & & & & & & & & & & & \\
\hline$\overline{R u f f e d}$ Grouse & & & & & & & & & 1 & & & & 5 & \\
\hline Sharp-tailed Grouse & & & & 2 & 5 & & & 1 & 9 & 15 & & & 5 & 3 \\
\hline Ring-neck. Pheasant & & & & & 9 & & 1 & & & 1 & & & & \\
\hline Gray Partridge & & 18 & & 37 & 15 & 18 & & 18 & & 43 & & & & \\
\hline American Coot & & & & & & & & & & & & & & \\
\hline Rock Dove & & & & 2 & $\pi$ & 1 & & 8 & & 281 & & & & \\
\hline Great-horned Owl & & 1 & & & & 1 & & & $\mathrm{I}$ & & & & $T$ & \\
\hline Snowy Owl & & & 1 & 2 & & 6 & & & & 1 & & & & \\
\hline Hawk Owl & & & & & & & & & & & & & & \\
\hline Great Gray Owl & & & & & & & & & & & 2 & & & \\
\hline Long-eared Owl & & & & & & & & & & & & & & \\
\hline Short-eared Owl & & & & & & & & & & & & & & \\
\hline Common Flicker & & & & & & & & & & & & & & \\
\hline Pileated Woodpecker & & & & & & & & & 1 & & & & & \\
\hline Hairy Woodpecker & 1 & & & & & & & & 2 & & & 1 & & 5 \\
\hline Downy Woodpecker & 1 & & & & & & & & 2 & $T$ & & & & 5 \\
\hline N. 3-t. Woodpecker & & & & & & & & & & & & & & \\
\hline Horned Lark & & & & 2 & 33 & & & & & & & & 2 & \\
\hline Gray Jay & 5 & & & & & & & & & & & & 2 & \\
\hline Blue Jay & 6 & & & & & & & & & & 1 & & $T$ & 5 \\
\hline Black-billed Magpie & 3 & 4 & 4 & 17 & 2 & 20 & 20 & 18 & 10 & 13 & 8 & & 4 & 42 \\
\hline Common Raven & $3 i$ & & & & & & & & & & 21 & & 1 & \\
\hline Bl.-cap. Chickadee & 2 & 2 & & & & & 2 & 5 & 17 & 9 & & & $\overline{9}$ & 12 \\
\hline Boreal Chickadee & & & & & & & & & & & & & & \\
\hline W-breasted Nuthatch & & & & & & & & & & & & & & 1 \\
\hline R-breasted Nuthatch & & & & & & & & & & & & & & \\
\hline Brown Creepcr & & & & & & & & & & & & & & \\
\hline Robin & & & & & & & & & & & & & & 1 \\
\hline Bohemian Waxwing & & & & & 19 & . & & 10 & & 60 & & 80 & & 625 \\
\hline Cedar Waxwing & & & & & & & & & & & & & & \\
\hline Northern Shrike & & & & & & 1 & & & 1 & & & & 1 & \\
\hline Starling & & & & & 3 & & & & & 70 & & & & \\
\hline House Sparrow & & 75 & 12 & 200 & 100 & 32 & 8 & 26 & 30 & 405 & 2 & & & 8 \\
\hline Rusty Blackbird & & & & & & & & & 1 & & & & & \\
\hline Brewer's Blackbird & & & & & & & & & & & & & & \\
\hline Common Grackle & & & & & & & & & & 1 & & & & \\
\hline Evening Grosbeak & & & & & & & & & & & 40 & & & \\
\hline Purple Finch & & & & & & & & & & & & & & 1 \\
\hline Pine Grosbeak & 2 & & & & & & & & 9 & 6 & $\mathrm{~T}$ & & 3 & 11 \\
\hline Hoary Redpoll & & & & 2 & & & & & 3 & & & & 1 & \\
\hline Common Redpoll & 47 & & & 25 & & & & 32 & 471 & 2 & 33 & & 158 & $\overline{13}$ \\
\hline Pine Siskin & & & & & & & & & & 12 & & & & \\
\hline W-winged Crossbill & & & & & & & & & & & & & & \\
\hline Dark-eyed Junco & & & & & & & & & & 1 & & & & \\
\hline Tree Sparrow & & & & & & & & & & & & & & \\
\hline W-throated Sparrow & & & & & & & & & & & & & & \\
\hline Snow Bunting & 3 & & & 59 & & 120 & & 1 & & 165 & & & 125 & $\overline{50}$ \\
\hline
\end{tabular}


ied-billed Grebe

Mute Swan

anada Goose

Mallard

Black Duck

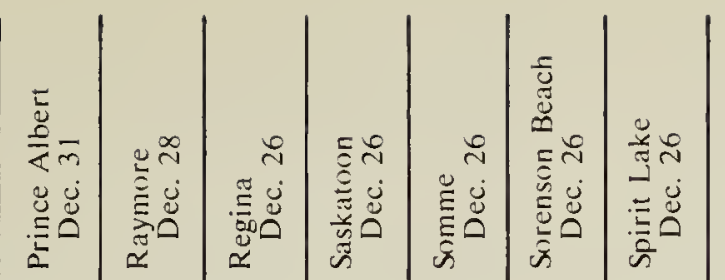

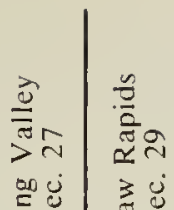

焉

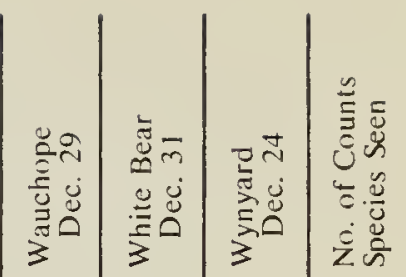

\section{iadwall}

intail

edhead

anvasback

esser Scaup

ommon Goldeneyc

ufflehead

uddy Duck

ommon Merganser

ed-br. Merganser

inshawk

ald Eagle

iyrfalcon

rairie Falcon

lerlin

uffed Grouse

harp-tailed Grouse

ing-neck. Phcasant

ray Partridge

merican Coot

ock Dove

reat-horned Owl

lowy Owl

awk Owl

reat Gray Owl

ong-eared Owl

ort-eared Owl

ommon Flicker

leated Wondpecker

airy Woodpecker

owny Woodpecker

3-t. Wondpecker

orned Lark

ray Jay

ue Jay

ack-billed Magpie

immon Raven

-cap. Chickadee

2

\section{real Chickadee}

-breasted Nuthatch

-breasted Nuthatch

fown Crecper

\begin{tabular}{|l|r|r|r|r}
\hline lhin & & & 9 & 6 \\
\hline lhemian Waxwing & 3 & 5 & 1034 & 1756 \\
\hline
\end{tabular}

edar Waxwing

irthern Shrike

arling

ouse Sparrow

Isty Blackbird

ewer's Blackbird

immon Grackle

ening Grosbeak

irple Finch

ne Grosbeak

ary Redpoll

mmon Redpoll

ne Siskin

-winged Crossbill

irk-eyed Junco

ee Sparrow

-throated Sparrow

ow Bunting

\begin{tabular}{r|r|r}
\hline & 2 \\
\hline & & 225 \\
\hline
\end{tabular}

\begin{tabular}{rr|r|r|r|r}
\hline & & & 500 & 45 & \\
\hline
\end{tabular}

\begin{tabular}{ll|l|}
\hline & & \\
\hline
\end{tabular}

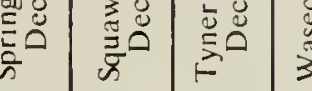

焉

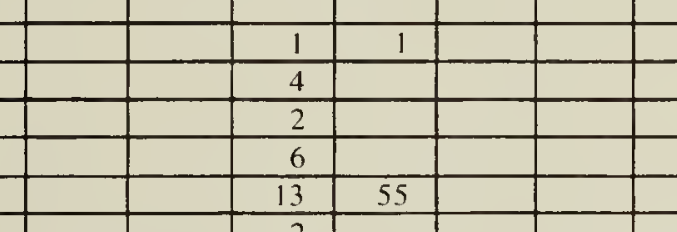

\begin{tabular}{|c|c|c|c|} 
& & & \\
& & & \\
& 11 & &
\end{tabular}


Joe Miller, Helen Morrison, David Phillips, John Pilling, Connic Pratt, Bob and Karen Rafuse, Brian Rainey, Susan Rockword, Diane Seeoy, Allan Smith, Frank Switzer, Bob Tegart, Dorothy Tegart, Elisabeth Wagner, Jeanie Wagner, Janie Wilhelm, Rita Wilhelm.

32. SASKATOON. Dee $26 ; 45 \mathrm{mi}$. in 46-1/2 hrs. ()n for)t, $227 \mathrm{mi}$. by ear in $34 \mathrm{hrs}$. and $15 \mathrm{mi}$. () $\mathrm{n}$ skis in $8-1 / 2$ hrs., temp. $17^{\circ}$ to $22^{\circ}$, wind W 10-15 mph, 15 in. snow: 39 species, 7474 individuals. (Add: Red Crossbill, White-winged Crossbill). Murray Akre, Moira Atkinson, Bob, Jeff and Joan Besant, Bernard, Ed, Raymond and Timothy Bisha, Ron Bobowski, André Bouthillette, Joseph E. Daly, Erie, Heather, Margot and Susan Diehl, Tom Donald, Alan and Hartley Fredeen, Betty and John Gerrard. J. B. Gollop, M. F. Gollop, Seott Hale, Danny Heffernan, Clarenee J. Houston, David, Donald, Mary, Stan and Stuart Houston, Shelley Laird, Dave and Richard Male, David and Pat Maloney, Jaeob Masligah, Don, Elizabeth, Joanne and Norman MeRobbie, Anna Miller, Alan Moulin, Arnold Nijssen, Lynn Oliphant, Stuart Rasmussen, Adam Sehmidt, David Schmidt, John and Stan Shadick, Jim Slimmon, Alan, Edward, Gary and Karen Smith, Peter Tassic, Michael Tyrrell, Sharon E. Wardle.

33. SOMME. Dee. 26; 3 hrs. on foot and 1 hr. by car for $35 \mathrm{mi}$., temp. $25^{\circ}$, calm; 13 species, 62 individuals. - Donald and Romald $\mathrm{Horper}$

34. SORENSON BEACH. Dec. 26; 3 hrs. by snowshoe, snowing, temp. 15\%; 11 species, 45 individuals. - Hanna and Heinz Ueberschar.

35. SPIRIT LAKE. Dec. 26: $2 \mathrm{mi}$. on foot in 2 hrs., $58 \mathrm{mi}$. by ear in 4 hrs. and $7 \mathrm{mi}$. in $1 \mathrm{hr}$. by snowmobile, temp. $20^{\circ}$, overeast with snow flurries, wind light, 13 in. snow; 18 species, 510 individuals. (Add: Great Horned Owl, Pileated Wordpecker, Common Raven, Evening Grosbeak, Snow Bunting). - Bill and Joyee Anaka.

36. SPRING VALLEY. Dee. 27; $28 \mathrm{mi}$. by car and $1-1 / 2 \mathrm{mi}$. on foot in 5 hrs., temp. $15^{\circ}$, overeast with light snow, wind 10-25 mph, 2-1/2 feet of snow; 10 species, 833 individuals. (Add: Great Horned Owl, 1 ,
Dec. 23: Rusty Blackbird, 1, Dec. 22: Com mon Redpoll, 15, Dee. 16). - Allan Bogdan, Larry Bogdan, Mr. and Mrs. Nick Bogdan, Gilberthal Liebelt.

37. SQUAW RAPIDS. Dee. 29; temp. $-20^{\circ}$ hoarfrost; 5 species, 29 individuals. - Stal and Gladys Riome.

38. TYNER. Dec. 28; $4 \mathrm{mi}$. on foot and 20 mi. by ear in 5 hrs., temp. $0^{\circ}$, wind 5 mph; 8 species, 75 individuals. - Bob Godwin Ken Godwin, Ken Lumbis (eompiler).

39. WASECA. Jan. 3; around yard and feeders: 11 species, 134 individuals. (Add Great Horned Owl). - Christine Pike.

40. WAUCHOPE. Dee. 29; 4-1/2 mi. by snowshoe in $3-1 / 2$ hrs., $35 \mathrm{mi}$. by ear in 2 . $1 / 2$ hrs., around farmyard for $1 \mathrm{hr}$. and 20 minutes, temp. $-20^{\circ}$ to $-10^{\circ}$, wind $W$ at 4 mph, elear, hoarfrost, 9 in. snow; 13 species, 387 individuals. - Dale Hjertaas 41. WHITE BEAR. Dec. 31: 2 mi. on foot and $10 \mathrm{mi}$. by car, temp. $-20^{\circ}$, clear, calm; 5 species, 117 individuals. (Add: Sharp-tailed Grouse, 6, Dee. 29; Common Redpoll, 20 Dec. 29). - Oran Cates, Leroy Clark, Mike Fowler, Gary Jordheim, Sig Jordhein (eompiler), Kenny Markulla, Danny Sehuler (Members of the Conservation Club).

42. WYNYARD. Dec. 24:57 mi. by car it 4-1/2 hrs., temp. 22\%, wind W $10 \mathrm{mph}$ overeast and some fog; 8 species, 570 in dividuals. - John and Sherry Gulley.

\section{N.W.T. CHRISTMAS BIRD COUNT}

FORT SMITH, N.W.T. Dec. 26; $50 \mathrm{mi}$. by ear and about the town of Fort Smith in 3 hours, temp. $-2^{\circ}$, overeast, ealm, 1 foot of snow; 7 species, 191 individuals, Willow Ptarmigan, 7; Rock Dove, 79; Gray Jay, 2: Common Raven, 65: House Sparrow, 14 Pine Grosbeak, 11; Common Redpoll, 13. (Add: Ruffed Grouse, 2, Dec. 24; Blackcapped Chickadee, 1, Dee. 27). - Elsie, Ernie, Jonathan and Pamela Kuyt, Sandra. Don, Jon-Liv Gaque, Brenda and Claire Beaulieu, Margaret Lepine.

The perfect book has been published by the Honolulu Zoo. The 20-page volume. "Snakes of Hawaii," is said to be "completely devoid of zoological, grammatical. and typographical errors." Small wonder! All the pages are completely blank. There are no snakes in Hawaii. Conservation News. March 1, 1974. 